\title{
Impacto del castor en la estructura de bosques ribereños de Nothofagus pumilio en Tierra del Fuego, Chile
}

\author{
Impact of beavers on Nothofagus pumilio riparian forest structure in Tierra del Fuego, Chile
}

\author{
Iván Arismendia, ${ }^{\mathrm{a}}$, Paul Szejnerc, Antonio Lara ${ }^{\mathrm{b}, \mathrm{c}}$, Mauro E. González ${ }^{\mathrm{b}, \mathrm{c}}$ \\ *Autor de correspondencia: aUniversidad Austral de Chile, Facultad de Ciencias Forestales, Valdivia, Chile, \\ ivanarismendi@uach.cl \\ bNúcleo Científico Milenio FORECOS, Valdivia, Chile. \\ 'Universidad Austral de Chile, Instituto de Silvicultura, Valdivia, Chile.
}

\begin{abstract}
SUMMARY
Beaver (Castor canadensis) is an exotic species that was introduced to Tierra del Fuego in 1946. Beaver dams have altered riparian areas existing before their invasion. This study evaluates the impact of beavers on the $N$. pumilio riparian forest structure in sites with and without beavers in two locations on Tierra del Fuego Island. At both sites, density, diametric distribution and basal area for alive and dead trees were measured. Most trees damaged by beavers were saplings or corresponded to a lower diameter class $(<25 \mathrm{~cm}, \mathrm{DBH})$. It was possible to observe that sites with beavers located in both sectors had individuals with significant lower diameter $(P<0.01)$. Also, sites with beavers had an individual mean density (Río Bueno 868 trees ha ${ }^{-1}$ and Vicuña 997 trees ha ${ }^{-1}$ ) higher than that from sites without beavers (Río Bueno 325 trees ha ${ }^{-1}$ and Vicuña 656 trees ha ${ }^{-1}$ ). This suggests that beavers differentially select invasion sites depending on the riparian forest structure, preferring places with high presence of saplings and trees with less than $25 \mathrm{~cm}$ diameter. One of the main impacts due to the establishment of beavers is the change in a diametric structure presented by stands in the riparian area. Due to the constant foraging from beavers, in the long-term an important alteration to the structure of the $N$. pumilio riparian forest in Tierra del Fuego may occur.
\end{abstract}

Key words: seedlings, beaver dams, secondary succession, regeneration.

\section{RESUMEN}

El castor es una especie exótica introducida en Tierra del Fuego en el año 1946. Sus represas construidas en los cursos de agua han modificado las zonas ribereñas existentes antes de su invasión. El presente estudio cuantificó el impacto del castor en la estructura del bosque ribereño de $N$. pumilio en lugares con y sin castores en dos sectores de la isla. En cada lugar se midieron la densidad, distribución diamétrica y área basal de árboles vivos y muertos. Los árboles muertos por el castor fueron, en su mayoría, brinzales y de DAP menor a $25 \mathrm{~cm}$. Se observó que lugares con presencia del castor presentaron individuos con un DAP significativamente menor en ambos sectores $(P<0,01)$. En lugares con presencia del castor la densidad promedio de individuos fue mayor (Río Bueno 868 árboles ha ${ }^{-1}$ y Vicuña 997 árboles $h^{-1}$ ) a la encontrada en lugares sin castores (Río Bueno 325 árboles ha ${ }^{-1}$ y Vicuña 656 árboles $\mathrm{ha}^{-1}$ ). Esto sugiere que el castor se establece diferencialmente de acuerdo a la estructura forestal del bosque ribereño y selecciona diferenciadamente los sitios de invasión, prefiriendo rodales con una abundante presencia de brinzales y árboles de clases diamétricas menores $(<25 \mathrm{~cm})$. Uno de los principales efectos de la invasión del castor es el cambio en la estructura diamétrica de los rodales presentes en la franja ribereña. De esta forma, por efecto del constante forrajeo, en el largo plazo puede ocurrir una importante alteración en la estructura del bosque ribereño de $N$. pumilio.

Palabras clave: brinzales, castoreras, sucesión secundaria, regeneración.

\section{INTRODUCCIÓN}

El castor del Canadá (Castor canadensis Kuhl) es una especie exótica e invasiva en Europa (Lathi y Helminen 1974) y Sudamérica (Godoy 1963) y su impacto ecológico requiere de un entendimiento diferente al de su hábitat nativo en Norteamérica (Baker y Hill 2003). Ello debido a su alta capacidad de invasión en un corto periodo de tiempo (Lizarralde 1993, Skewes et al. 2006). En Sudamérica, la invasión del castor se inició en 1946 con la liberación de 25 parejas en el lado argentino de la Isla Grande de Tierra del Fuego (Lizarralde et al. 2004). Actualmente se encuentra presente en gran parte del archipiélago fueguino y se estima, sólo en el territorio chileno, una población de más de 61.000 individuos (Skewes et al. 2006). La ausencia de predadores naturales y competidores aumenta su capacidad de invasión, lo que se traduce en una amenaza hacia el continente debido a su potencial establecimiento en todos los bosques templados de Sudamérica entre los $35^{\circ}-55^{\circ} \mathrm{S}$ (Wallem et al. 2007). 
El castor es considerado un ingeniero ecosistémico (Jones et al. 1994, Crooks 2002) debido a que, por efecto de la construcción de represas (castoreras) en los cursos de agua en que habita, se crean nuevos hábitats que son utilizados tanto por ellos como por el resto de la flora y fauna existente en el área (Baker y Hill 2003). En ambientes terrestres de su distribución original en el hemisferio norte, los impactos que provoca el castor en la estructura y composición de la vegetación ribereña son bien conocidos (Ives 1942, Naiman et al. 1994, Donkor y Fryxell 1999, Wright et al. 2002). Los impactos directos son derivados del corte de árboles para forrajeo y construcción de diques (Baker y Hill 2003), los cuales provocan cambios en la estructura y composición del bosque (Naiman et al. 1994, Donkor y Fryxell 1999). El castor prefiere utilizar árboles de menor diámetro, lo que causa una disminución de la densidad y área basal en el área cercana a las castoreras (Johnston y Naiman 1990, Barnes y Mallik 1996). Dicha preferencia se ha descrito como un mecanismo importante de facilitación para el establecimiento de plántulas de algunas especies arbóreas (Cottrell 1995), lo que puede alterar la sucesión forestal natural con el reemplazo de especies deciduas por coníferas (Donkor y Fryxell 1999).

Los impactos indirectos resultan de la modificación del suelo y geomorfología ribereña una vez abandonada la castorera y destruido el dique debido a la creación de embalses colmatados de sedimentos (Baker y Hill 2003). Con el tiempo, dichos embalses forman meadows, definidos como hábitats ocupados totalmente por pastizales y plantas no arbustivas (Ives 1942, Naiman et al. 1994), donde se establece un ensamble característico de especies herbáceas (Wright et al. 2002) y la sucesión forestal ocurriría de una forma mucho más lenta de lo esperado (Baker y Hill 2003). Dicho retardo se asociaría a mecanismos ecológicos complejos e interacciones específicas entre raíces y micorrizas (Terwilliger y Pastor 1999).

En Sudamérica, los impactos indirectos del castor provocados por la generación de meadows se han comenzado a estudiar recientemente (Anderson et al. 2006, MartínezPastur et al. 2006). Anderson et al. (2006), en un estudio en la Isla Navarino al sur de Tierra del Fuego, indican que en los meadows ocurre un activo reclutamiento de plantas, aumentando la riqueza de especies herbáceas nativas y exóticas. Sin embargo, dichos ensambles no son distintos a los encontrados en áreas boscosas. A su vez, MartínezPastur et al. (2006) muestran que en meadows abandonados por más de 20 años existiría un impedimento o retardo en la regeneración del bosque ribereño de Nothofagus spp. Sin embargo, existe aún desconocimiento y falta de evidencia empírica sobre los impactos directos del castor en la estructura del bosque ribereño, especialmente en los bosques dominados por lenga (Nothofagus pumilio (Poep. et Endl) Krasser). Sólo un estudio realizado por Lizarralde (1993) en Tierra del Fuego da cuenta de la estructura diamétrica de troncos y ramas utilizados por el castor, encontrándose mayores diámetros usados en las zonas cercanas a los diques y menores diámetros en las zonas más alejadas.

El objetivo de este trabajo es contribuir con evidencia empírica que permita cuantificar el impacto directo del castor en la estructura del bosque ribereño de lenga en dos sectores de la Isla Grande de Tierra del Fuego. Se pretende así ayudar al entendimiento del patrón de invasión e influencia de esta especie en la dinámica de los bosques subantárticos. La hipótesis de trabajo es que el castor, al igual que en el hemisferio norte, selecciona árboles de menor diámetro, mostrando una preferencia por lugares que presentan una determinada estructura diamétrica de bosque ribereño.

\section{MÉTODOS}

Área de estudio. Los sitios de muestreo se localizaron en la parte central de la Isla Grande de Tierra del Fuego, en la Reserva Karukinka, sectores Río Bueno y Vicuña, en un rango de altitud entre 150 y $312 \mathrm{~m}$ s.n.m. (figura 1). Fisiográficamente esta zona se denomina subandina oriental de clima trasandino con degeneración esteparia (Pisano 1977), presentando un rango anual de precipitaciones entre 338 y $852 \mathrm{~mm}$, y una temperatura media anual entre 2,7 y $5,3^{\circ} \mathrm{C}$ (Henríquez 2004). Ambos sectores son representativos de los bosques subantárticos costeros (Río Bueno) y de aquellos de transición hacia la estepa (Vicuña). En el sector Río Bueno, se encuentran bosques con predominancia

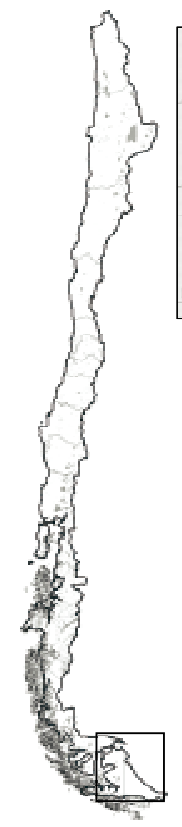

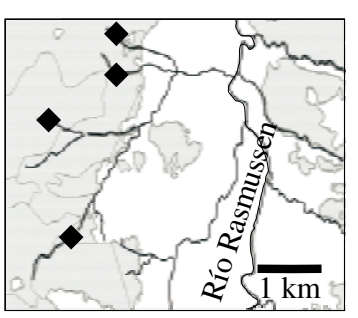

囚 Sector Vicuña

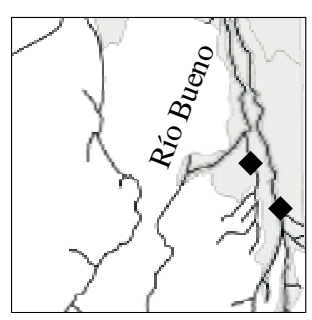

\ Sector Río Bueno

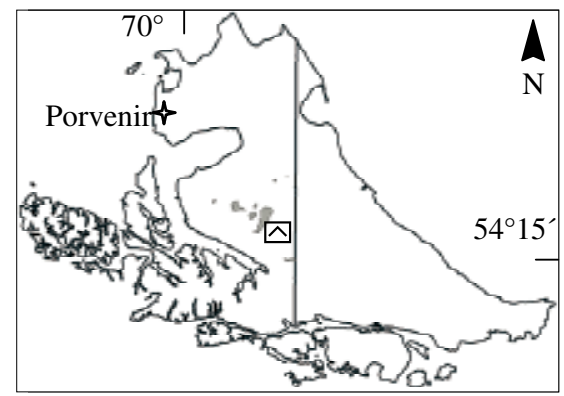

Isla Grande de Tierra del Fuego

Figura 1. Área de estudio dentro de la Isla Grande de Tierra del Fuego indicando los arroyos muestreados en cada sector (Vicuña y Río Bueno). El área sombreada representa el uso de suelo cubierto por bosque de lenga.

Study area on the big island of Tierra del Fuego indicating the sampled streams for each sector (Río Bueno and Vicuña). Shadowed area represents the land use covered by lenga forest. 
de los tipos forestales lenga y en menor proporción coigüe de Magallanes (Nothofagus betuloides (Mirb) Oerst.), mientras que en el sector Vicuña existen ambientes mixtos de estepa húmeda, turbales y bosques de lenga (Mella 1994). Además, en ambos sectores, la invasión del castor se ha descrito como ocurrida desde principios de los años 1970 (Skewes et al. 2006).

Muestreo en terreno. Para caracterizar la estructura del bosque ribereño se eligieron seis arroyos de segundo orden, de los cuales cuatro se ubicaron en el sector Vicuña y dos en el sector Río Bueno (figura 1). A su vez, en cada uno de los arroyos se exploró su ribera e identificó, previo al muestreo, los lugares con y sin impacto del castor (figura 2). Los lugares con impacto del castor se definieron como tales cuando presentaron la existencia de diques y castoreras activas, y la presencia de marcas del castor en el bosque (árboles forrajeados y corteza dañada por sus dientes). Por su parte, los lugares sin impacto fueron aquellos que no presentaron ninguno de los atributos mencionados anteriormente, y donde no se encontró evidencia alguna de la existencia de castoreras antiguas. Luego de ello, en cada lugar se definieron unidades muestreales correspondientes a parcelas de $20 \times 10 \mathrm{~m}$ medidas a $1 \mathrm{~m}$ de distancia desde el borde del arroyo (o embalse para el caso de las castoreras) y separadas cada $50 \mathrm{~m}$ entre sí a lo largo de un transecto paralelo al curso de agua. En el caso de los sitios con impacto del castor, los transectos se iniciaron a la altura del dique. Se establecieron un total de 62 parcelas, 17 en el sector Río Bueno y 45 en el sector Vicuña. En cada parcela fueron contabilizados todos los individuos vivos y muertos, y cuyo eje central estaba dentro de la parcela. A cada individuo se le midió el diámetro a la altura del tocón (DAT) y diámetro normal a 1,3 m (DAP). A los árboles forrajeados por el castor y otros que sólo presentaron tocones se les estimó el DAP de acuerdo a lo propuesto por Bava y López-Bernal (2006). Se denominó brinzal a los individuos con DAP menor o igual a $5 \mathrm{~cm}$ y mayor a $2 \mathrm{~m}$ de altura. Además, en cada parcela se estimó la densidad de individuos (D, individuos ha ${ }^{-1}$ ) y el área basal $\left(\mathrm{G}, \mathrm{m}^{2} \mathrm{ha}^{-1}\right)$, la que se definió como:

$$
G=D \times \frac{\Pi}{40.000} \sum_{i=1}^{j} C D A P_{i}^{2} \times F r_{i}
$$

Donde:

$\mathrm{G} \quad=$ Área basal de la parcela $\left(\mathrm{m}^{2} \mathrm{ha}^{-1}\right)$.

$\mathrm{D}=$ Densidad promedio de individuos en cada parcela.

$\mathrm{CDAP}_{\mathrm{i}}=$ Valor central de la clase diamétrica DAP (agrupada en intervalos cada $5 \mathrm{~cm}$, desde la clase i hasta la j-ésima)

$\mathrm{Fr}_{\mathrm{i}} \quad=$ Frecuencia relativa de cada clase diamétrica (desde la clase i hasta la j-ésima).
Posteriormente, se estimó el diámetro medio cuadrático (DMC), definido como el diámetro equivalente al árbol de área basal media. Se identificaron además los individuos vivos con cicatrices en el fuste causadas por mordeduras de castor, y tocones o árboles muertos derivados del impacto directo del castor. El muestreo se realizó durante el verano del año 2007.

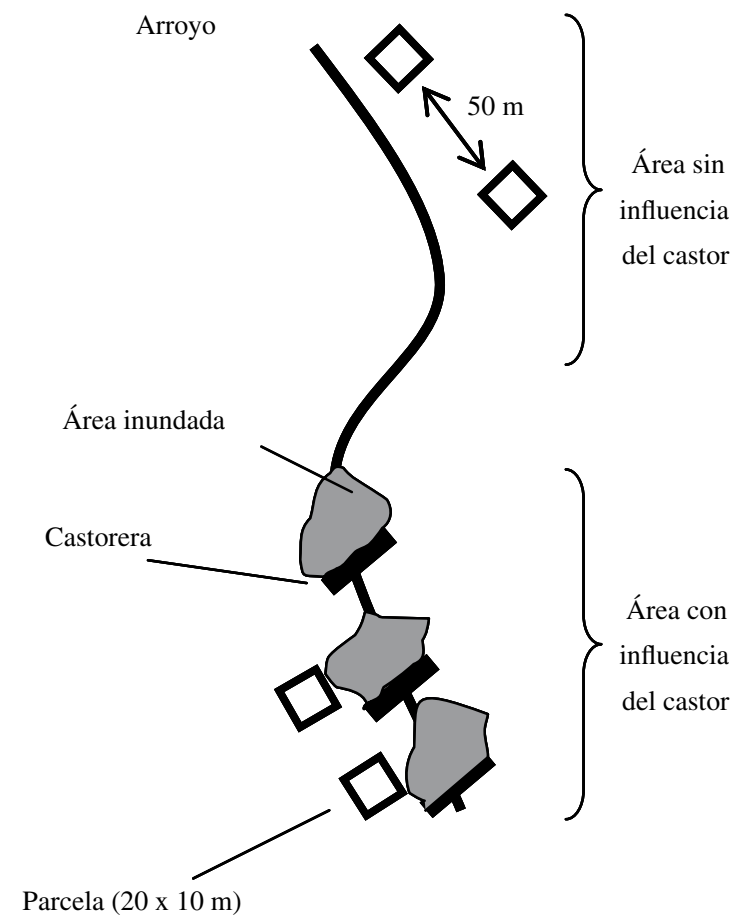

Figura 2. Diseño de muestreo indicando la ubicación de las parcelas de 20 x 10 (m), en lugares con y sin impacto del castor, dentro de cada arroyo muestreado.

Experimental design showing the location of plots $(20 \times 10$ $\mathrm{m})$ in sites with and without beavers for each sampled stream.

Análisis estadístico. Con objeto de comparar el DAP, DMC y densidad de individuos por parcela, entre sectores (Río Bueno y Vicuña) y lugares (con y sin influencia del castor), se utilizó un ANDEVA factorial con un nivel de confianza del 95\%, considerando como factores los lugares (con y sin castores) y sectores (Río Bueno y Vicuña). Los resultados se presentaron como promedio \pm error estándar. En el caso de la densidad se cumplieron los supuestos de normalidad y homogeneidad de varianzas a través de la transformación $y=\operatorname{Ln}($ densidad +1$)$. En la comparación de la estructura diamétrica del bosque ribereño los individuos vivos y muertos por acción directa del castor se consideraron en una misma categoría ya que no fue posible determinar la data exacta de muerte. Por lo tanto, es necesario considerar un cierto error en la clasificación de estos individuos en una determinada clase diamétrica -ubicados en clases menores debido a la detención de su crecimiento- dependiendo de su fecha de muerte. De acuerdo a esto, se puede inferir que el máximo error ocurrió en la clasificación de los individuos 
que murieron durante el inicio de la invasión del castor, es decir, hace 37 años aproximadamente (Skewes et al. 2006). Entonces, y considerando una tasa de crecimiento en diámetro descrita para lenga en el sur de la Patagonia de 0,15-0,19 cm año ${ }^{-1}$ (Rubilar 1991, Rebertus y Veblen 1993, Aravena et al. 2002, Cruz et al. 2007), se estimó un error máximo posible de 5,5 a 7,0 cm de diámetro. A su vez, de acuerdo a la amplitud de clase diamétrica utilizada en el análisis $(5-10 \mathrm{~cm})$, los individuos muertos podrían estar desplazados, como máximo, sólo en una clase diamétrica bajo la cual les correspondería situarse si estuvieran vivos.

Para comparar el área basal de parcelas con y sin impacto del castor se utilizó una prueba de t-Student con un nivel de confianza del $95 \%$. Además, con objeto de comparar las distribuciones del área basal por clase diamétrica se utilizó un análisis de bondad de ajuste de Chi-cuadrado, donde las frecuencias esperadas correspondieron a la situación sin impacto del castor y las observadas a la situación con impacto del castor. Similar análisis se realizó para comparar la distribución del número de parcelas por clase de DMC en lugares con y sin castores. Ello con objeto de presentar la dispersión de los individuos de menor diámetro dentro del número total de parcelas y descartar la presencia de sólo un par de parcelas con muchos individuos de menor diámetro (DAP) que pudieren provocar un sesgo en los resultados. Todos los análisis estadísticos se realizaron utilizando el software Statistica 6.0 software (StatSoft Inc., Tulsa, Oklahoma, USA).

Como supuesto del trabajo se asumió que todos los árboles menores a $5 \mathrm{~cm}$ y menores a $2 \mathrm{~m}$ de altura (brinzales) tienen más de 35-40 años de edad, y que se establecieron previos a la invasión del castor. Dicho criterio se sustentó, por una parte, en lo encontrado por Rebertus y Veblen (1993) en bosques puros de lenga en la parte argentina de Tierra del Fuego, donde se estimó que después de 35-40 años los individuos alcanzaron los dos metros de altura. Ello además concuerda con el estudio realizado por Cruz et al. (2007) en la Reserva Nacional Magallanes, donde se indica que individuos que alcanzaban un DAP de $5 \mathrm{~cm}$ correspondían a árboles de 40 años.

\section{RESULTADOS}

Tanto en Río Bueno como en Vicuña el bosque ribereño se compuso en un $100 \%$ de lenga. Del total de individuos medidos en Río Bueno y Vicuña $(\mathrm{n}=971)$, un 39\% no presentó evidencia de impacto provocado por el castor; sin embargo, en ambos sectores la proporción de individuos muertos por efecto directo del castor fue mayor a la proporción de los dañados (figura 3). A su vez, en ambos sectores, la distribución de individuos muertos por efecto directo del castor correspondió en su mayoría a brinzales y árboles menores a $25 \mathrm{~cm}$ DAP (más del $75 \%$ en ambos sectores). Sin embargo, los árboles que evidenciaron daño del castor, por presencia de cicatrices en la corteza, fueron en gran proporción individuos con un DAP mayor a $20 \mathrm{~cm}$.

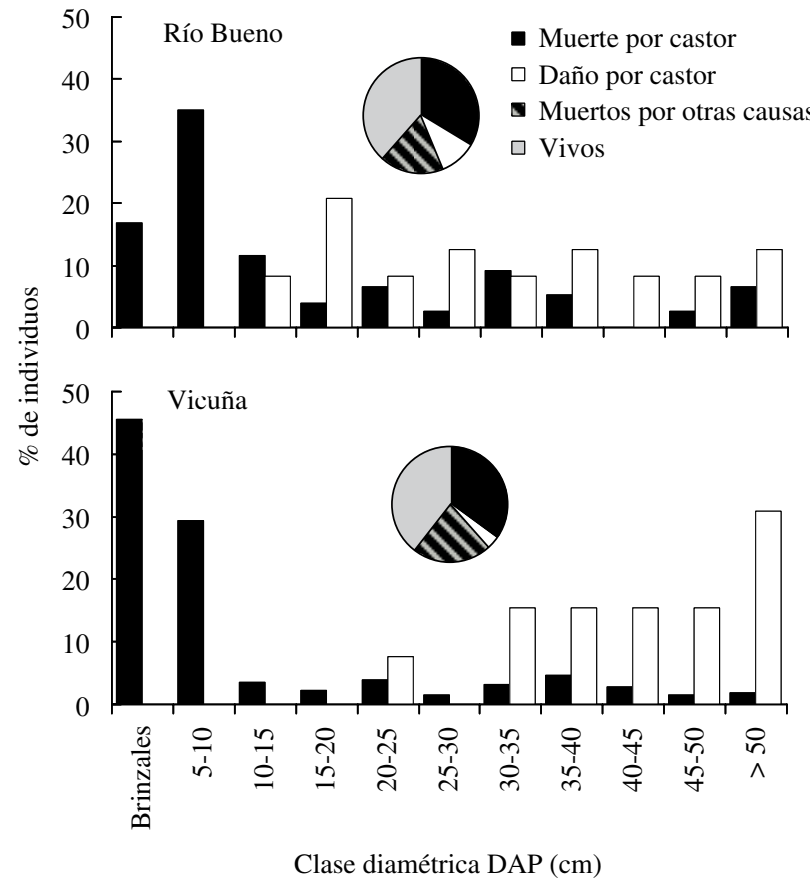

Figura 3. Porcentaje de individuos muertos y dañados por el castor agrupados por clase diamétrica (DAP) para los sectores Río Bueno $\left(\mathrm{n}_{\text {muertos }}=57, \mathrm{n}_{\text {dañados }}=44\right)$ y Vicuña $\left(\mathrm{n}_{\text {muertos }}=224\right.$, $\mathrm{n}_{\text {dañados }}=61$ ). Para cada sector, del total de individuos medidos $\left(\mathrm{n}_{\text {Bueno }}=230, \mathrm{n}_{\text {Vicuña }}=741\right)$, se incluye la proporción de árboles vivos, impactados por el castor (muertos y dañados), y muertos por otras causas no determinadas.

Percentage of individuals killed and damaged by beavers grouped by diametric class (DBH) in the Río Bueno $\left(\mathrm{n}_{\text {death }}=57\right.$, $\left.\mathrm{n}_{\text {damage }}=44\right)$ and Vicuña $\left(\mathrm{n}_{\text {death }}=224, \mathrm{n}_{\text {damage }}=61\right)$ sectors. For each sector and from all individuals measured, $\left(\mathrm{n}_{\text {Bueno }}=230, \mathrm{n}_{\text {Vicuña }}=741\right)$ the proportion of alive trees, individuals impacted by beavers (dead and damaged) and dead due to other undetermined reasons are included.

En lugares con presencia del castor, los brinzales (vivos y muertos por causa directa del castor) estuvieron presentes en un $11 \%$ de las parcelas muestreadas, mientras que los de la clase diamétrica inmediatamente superior ocurrieron en el $16 \%$ de ellas (cuadro 1). Para el caso de los lugares sin castores, los brinzales estuvieron presentes sólo en una parcela, y la clase inmediatamente superior en un $9,6 \%$ respecto del total de parcelas muestreadas. Por su parte, al agrupar ambas clases diamétricas (brinzales y clase 5-10 cm), la densidad promedio en lugares con castores fue de 265 árboles $\mathrm{ha}^{-1}$, mientras que en lugares sin castores fue menor (192,8 árboles ha-1). Al mismo tiempo, la distribución del número total de parcelas agrupadas por DMC, en lugares con y sin castores (DMC, cuadro 2), fue significativamente distinta $\left(\chi_{8}^{2} ; P<0,05\right)$. En lugares con presencia del castor, más del $45 \%$ de las parcelas presentaron un DMC inferior a $50 \mathrm{~cm}$; en lugares sin castor, en 
cambio, más del $90 \%$ de las parcelas presentaron un DMC superior a $50 \mathrm{~cm}$.

Cuadro 1. Parcelas donde se encontraron brinzales e individuos de la clase diamétrica 5-10 cm (DAP) en lugares con y sin castor. Se incluye la densidad de individuos vivos y muertos (por efecto directo del castor).

Plots with saplings and individuals in the $5-10 \mathrm{~cm}(\mathrm{DBH})$ diametric class in sites with and without beavers. The density of alive and dead individuals (caused by direct impact from beavers) is included.

\begin{tabular}{|c|c|c|c|c|c|}
\hline \multirow{3}{*}{ Lugar } & \multirow{3}{*}{ Parcela } & \multicolumn{4}{|c|}{ Densidad (individuos $\mathrm{ha}^{-1}$ ) } \\
\hline & & \multicolumn{2}{|c|}{ Vivos } & \multicolumn{2}{|c|}{ Muertos por castor } \\
\hline & & Brinzales & $5-10 \mathrm{~cm}$ & Brinzales & $5-10 \mathrm{~cm}$ \\
\hline \multirow{10}{*}{$\begin{array}{l}\text { Con } \\
\text { impacto } \\
\text { del } \\
\text { castor }\end{array}$} & 1 & - & 200 & 50 & 150 \\
\hline & 2 & - & 100 & 100 & 200 \\
\hline & 3 & - & - & - & 150 \\
\hline & 4 & - & 150 & - & 200 \\
\hline & 5 & - & 50 & - & 200 \\
\hline & 6 & - & 150 & 250 & 550 \\
\hline & 7 & 50 & - & 900 & 700 \\
\hline & 8 & - & - & 350 & 1.150 \\
\hline & 9 & - & - & 750 & 850 \\
\hline & 10 & - & - & 2.600 & 2.250 \\
\hline \multirow{6}{*}{$\begin{array}{l}\text { Sin } \\
\text { impacto } \\
\text { del } \\
\text { castor }\end{array}$} & 1 & - & 100 & - & - \\
\hline & 2 & - & 50 & - & - \\
\hline & 3 & - & 50 & - & - \\
\hline & 4 & - & 50 & - & - \\
\hline & 5 & 450 & 600 & - & - \\
\hline & 6 & - & 50 & - & - \\
\hline
\end{tabular}

Cuadro 2. Distribución del número de parcelas agrupadas por intervalos de DMC de individuos vivos y muertos (por efecto directo del castor), para lugares con y sin castor. Los lugares sin castor fueron considerados como frecuencias esperadas, mientras que los lugares con castor se consideraron como frecuencias observadas (Chi-cuadrado $=21,6 ; \mathrm{gl}=8 ; P<0,05$ ).

Distribution of the number of plots grouped by intervals of QMD for alive and dead individuals (caused by direct impact from beavers) in sites with and without beavers. Sites without beavers were considered as expected frequencies and sites with beavers were considered as observed frequencies $($ Chi-Square $=21.6, \mathrm{df}=8, P<0.05)$.

\begin{tabular}{ccc}
\hline \multirow{2}{*}{$\begin{array}{c}\text { Clase DMC }(\mathrm{cm}) \\
\text { vivos + muertos }\end{array}$} & $\begin{array}{c}\text { Sin castor } \\
\text { (frecuencias } \\
\text { esperadas) }\end{array}$ & $\begin{array}{c}\text { Con castor } \\
\text { (frecuencias } \\
\text { observadas) }\end{array}$ \\
\hline $10-20$ & 0 & 2 \\
$20-30$ & 0 & 2 \\
$30-40$ & 1 & 3 \\
$40-50$ & 2 & 7 \\
$50-60$ & 12 & 8 \\
$60-70$ & 9 & 6 \\
$70-80$ & 3 & 2 \\
$80-90$ & 2 & 0 \\
$90-100$ & 1 & 0 \\
\hline
\end{tabular}

Al comparar la estructura diamétrica (DAP) de los individuos vivos y muertos (por efecto directo del castor) por parcela, se encontraron diferencias significativas entre lugares con y sin castores $\left(\mathrm{F}_{1,58}=10,83 ; P<0,01\right)$. De esta forma, el DAP fue menor en lugares con presencia del castor, tanto en Vicuña $(19,3 \pm 3,1 \mathrm{~cm})$ como en Río Bueno $(20,8 \pm 3,6 \mathrm{~cm})$, en comparación a lugares sin castores (36 $\pm 2,5$ y $45,7 \pm 5,1 \mathrm{~cm}$, respectivamente). Dichas diferencias no fueron significativas entre sectores (Río Bueno y Vicuña: $\left.\mathrm{F}_{1,58}=0,06, P=0,80\right)$ y en la interacción lugar-sector $\left(\mathrm{F}_{1,58}=1,35 ; P=0,25\right)$. Adicionalmente, en el caso del DMC (incluyendo individuos vivos y muertos por efecto directo del castor) también se encontraron diferencias significativas entre lugares con y sin castores $\left(\mathrm{F}_{1,58}=11,19\right.$; $P<0,001)$, es así que tanto en Vicuña como en Río Bueno el DMC fue menor en lugares con presencia del castor (cuadro 3).

Cuadro 3. Distribución del número de parcelas y el DMC de individuos vivos y muertos (por efecto directo del castor) en cada sector (Río Bueno y Vicuña) y lugar (con y sin impacto del castor). ES representa el error estándar.

Distribution of the number of plots and QMD for alive and dead individuals (caused by direct impact from beavers) in each sector (Río Bueno and Vicuña) and site (with and without beavers). ES is the standard error.

\begin{tabular}{lrcc}
\hline Sector/Lugar & $\mathrm{N}^{\circ}$ parcelas & DMC promedio $(\mathrm{cm})$ & $\mathrm{ES}$ \\
\hline Río Bueno & & & \\
$\quad$ Con castor & 11 & 33,4 & 3,6 \\
$\quad$ Sin castor & 6 & 49,3 & 5,9 \\
Vicuña & & & \\
Con castor & 22 & 35,4 & 2,8 \\
Sin castor & 23 & 43,6 & 2,2 \\
\hline
\end{tabular}

En relación a la densidad promedio por parcela (individuos vivos y muertos por efecto directo del castor), se encontraron diferencias significativas entre lugares con y sin castores $\left(\mathrm{F}_{1,58}=10,04 ; P<0,01\right) \mathrm{y}$ entre los sectores Río Bueno y Vicuña $\left(\mathrm{F}_{1,58}=5,54 ; P<0,05\right)$, no así en la interacción lugar/sector $\left(\mathrm{F}_{1,58}=2,73 ; P=0,10\right)$. De esta forma, lugares con presencia del castor tuvieron una densidad promedio de individuos mayor (Río Bueno $868 \pm 174$ árboles ha ${ }^{-1}$ y Vicuña $997 \pm 224$ árboles ha $\left.{ }^{-1}\right)$ a la encontrada en lugares sin castores (Río Bueno $325 \pm 59$ árboles ha ${ }^{-1}$ y Vicuña $656 \pm 71$ árboles ha ${ }^{-1}$ ). Asimismo, la densidad promedio por parcela agrupada por clase diamétrica (incluyendo todos los individuos vivos y muertos) en ambos sectores (figura 4) mostró que lugares sin castores presentaron clases modales mayores a lo ocurrido en lugares con castores. Para los lugares sin castores la moda fue la clase $35-40 \mathrm{~cm}$, mientras que en lugares con presencia del castor, las clases modales fueron brinzales y clase diamétrica inmediatamente superior, en Vicuña y Río Bueno respectivamente. Adicionalmente, en lugares 


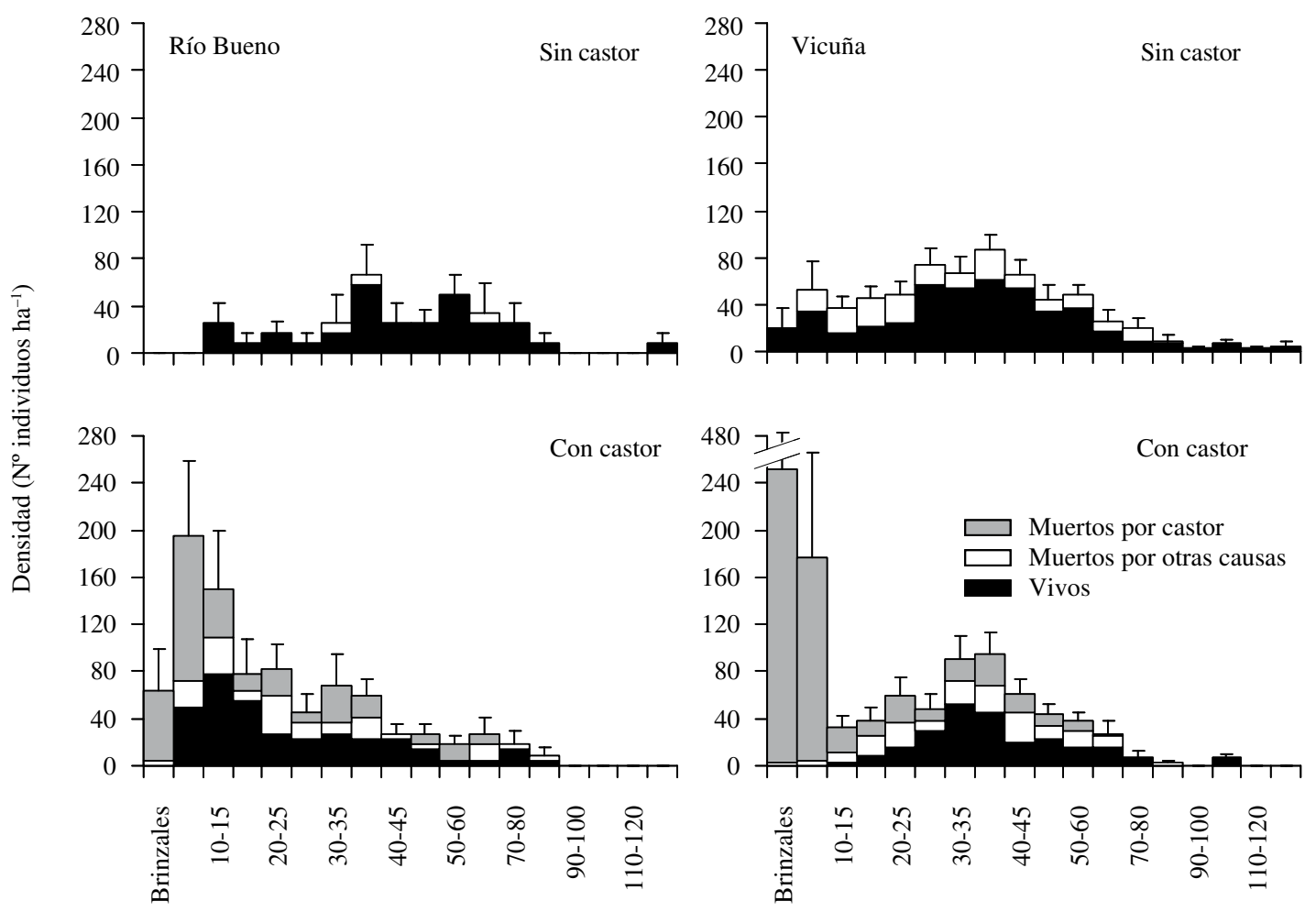

Clase diamétrica DAP $(\mathrm{cm})$

Figura 4. Distribución de la densidad de individuos (promedio + ES) por clase diamétrica (DAP) para los sectores Río Bueno y Vicuña en parcelas con y sin impacto del castor. Se incluyen los individuos vivos, muertos por causa directa del castor, y muertos por otras causas no identificadas. Clases diamétricas desde brinzales hasta $50 \mathrm{~cm}$ agrupadas cada $5 \mathrm{~cm}$, las mayores a $50 \mathrm{~cm}$ agrupadas cada $10 \mathrm{~cm}$.

Distribution of the density of individuals (mean + SE) grouped by diametric class (DBH) in Río Bueno and Vicuña sectors for plots with and without beavers. Alive and dead individuals killed by beavers, and dead individuals killed by other causes were included. Classes from stands up $50 \mathrm{~cm}$ were grouped by $5 \mathrm{~cm}$, from others above $50 \mathrm{~cm}$ were grouped by $10 \mathrm{~cm}$.

sin castores, las densidades máximas por clase diamétrica no sobrepasaron los 100 individuos $\mathrm{ha}^{-1}$, mientras que en sitios con castores algunas clases de menor diámetro presentaron densidades casi el doble de lo ocurrido en lugares sin castores.

Al comparar el área basal de individuos vivos y muertos (por efecto directo del castor) por parcela, se encontraron diferencias significativas entre lugares con y sin castores $\left(\mathrm{t}_{60}=2,31 ; P<0,05\right)$. Los mayores valores de área basal se encontraron en sitios sin castores, tanto para Vicuña $\left(87,5 \pm 5,7 \mathrm{~m}^{2} \mathrm{ha}^{-1}\right)$ como para Río Bueno $(65,8 \pm 16,5$ $\left.\mathrm{m}^{2} \mathrm{ha}^{-1}\right)$, en comparación a lugares con castores $(69,8 \pm 6,1$ y $57,5 \pm 7,1 \mathrm{~m}^{2} \mathrm{ha}^{-1}$, respectivamente). Finalmente, en la comparación de la distribución del área basal por clase diamétrica de individuos vivos y muertos (por efecto del castor) para lugares con y sin la presencia del castor (figura 5), se encontraron diferencias estadísticamente significativas sólo en el sector Río Bueno $\left(\chi_{23}^{2} ; P<0,001\right)$.

\section{DISCUSIÓN}

Este trabajo presenta evidencia del comportamiento selectivo del castor hacia el uso preferente de dos grupos de árboles con diámetros distintos en el caso de bosques dominados por lenga. El primer grupo estaría compuesto por brinzales y árboles de diámetro menor a $25 \mathrm{~cm}$, donde su consumo resulta en la muerte de dichos individuos ya que rara vez tienen la capacidad de rebrotar. El segundo grupo correspondería a individuos de mayor diámetro, los que en su mayor proporción serían dañados en su corteza sin provocarles necesariamente la muerte. El corte, generalmente a nivel basal, que realiza el castor provoca la muerte de los individuos de menor diámetro, lo que está dado por sus hábitos alimenticios de corteza y cambium de especies leñosas, además de hojas y ramillas (Baker y Hill 2003). Además, dichos individuos de menor diámetro serían principalmente utilizados -debido a su fácil transporte- 


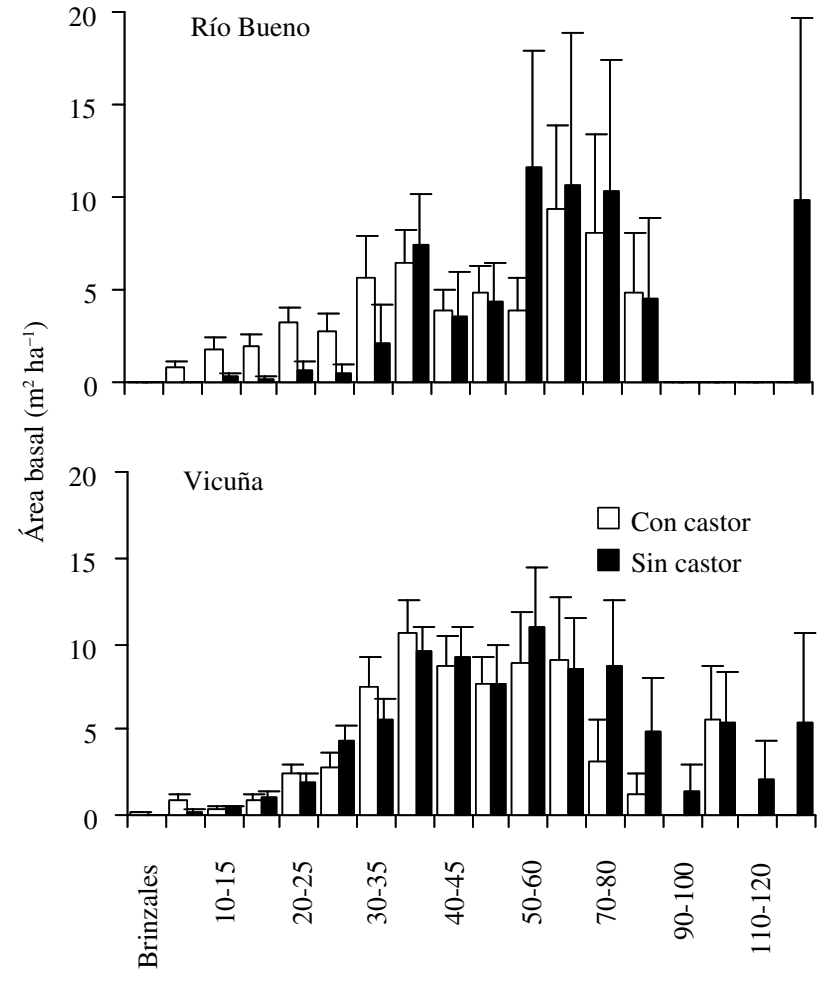

Clase diamétrica DAP $(\mathrm{cm})$

Figura 5. Distribución del área basal (promedio + ES) por clase diamétrica (DAP) de individuos vivos y muertos (por efecto directo del castor) para los sectores Río Bueno y Vicuña. Se incluyen parcelas con y sin impacto del castor. Clases diamétricas desde brinzales hasta $50 \mathrm{~cm}$ agrupadas cada $5 \mathrm{~cm}$, las mayores a 50 $\mathrm{cm}$ agrupadas cada $10 \mathrm{~cm}$.

Distribution of basal area (mean $+\mathrm{SE}$ ) grouped by diametric class (DBH) for alive and dead individuals (caused by direct impact from beavers) in Río Bueno and Vicuña sectors. Classes from stands up $50 \mathrm{~cm}$ were grouped by $5 \mathrm{~cm}$, from others above $50 \mathrm{~cm}$ were grouped by $10 \mathrm{~cm}$.

para la construcción de las represas o castoreras. En el caso de los individuos de mayor diámetro $(>20 \mathrm{~cm})$, una porción de ellos sería cortada y utilizada para dar el soporte estructural de algunas castoreras. Sin embargo, la mayor proporción de los árboles de mayor diámetro estaría siendo afectada por el daño provocado al fuste asociado a la necesidad permanente de los castores por desgastar sus dientes que biológicamente crecen durante toda su vida (Baker y Hill 2003).

Los resultados del presente estudio muestran evidencia sustancial de que lugares con presencia del castor, independientemente del sector en que ocurra (Río Bueno o Vicuña), presentan una densidad de individuos mayor y un diámetro menor (DAT, DMC) que lo observado en lugares sin castores. En estos últimos, donde ocurre una menor densidad de individuos pero con un mayor diámetro, finalmente resulta un área basal mayor. Esto sugiere que el castor selecciona diferencialmente los sitios de invasión, prefiriendo rodales con individuos de menor diámetro y probablemente también menor edad, y que corresponderían a estados sucesionales intermedios (o más avanzados con presencia de claros) con abundante presencia de brinzales. Es así que los lugares con una alta densidad de brinzales, como Vicuña, serían los preferidos por el castor, ya que precisamente lugares con baja abundancia de brinzales no fueron invadidos (figura 4). Briones et al. (2001), en Tierra del Fuego, encontraron que en la elección del hábitat a invadir por parte del castor las características topográficas de microcuenca serían las más relevantes a considerar, sin embargo, de los resultados del presente trabajo se desprende que la disponibilidad de brinzales sería clave en dicha elección. Los resultados del presente estudio concuerdan con lo que ocurre en el hemisferio norte, donde la estructura y composición de la vegetación ribereña puede influenciar la distribución del castor (Howard y Larson 1985, Barnes y Mallik 1997). Además, también se ha determinado una preferencia del castor por individuos de diámetros pequeños $(1,5-3,5 \mathrm{~cm})$, lo que resulta posteriormente en la disminución de la densidad y el área basal en sitios cercanos a las castoreras (Johnston y Naiman 1990, Barnes y Mallik 1996). En este caso, si se consideran sólo los individuos vivos, se puede constatar dicha disminución en la densidad y área basal luego de la invasión (figura 4).

Las diferencias encontradas en cuanto la densidad de árboles y área basal entre sectores (Río Bueno y Vicuña) serían explicadas por la variabilidad propia asociada al estado de desarrollo de los rodales, diferencias de sitio e historia de disturbios que modelan los bosques de lenga en Tierra del Fuego (Rebertus y Veblen 1993, Rebertus et al. 1997). De esta forma, en el momento de la invasión del castor, probablemente tanto Río Bueno como Vicuña mantenían dos estructuras de bosque distintas. Ello se sustenta en que típicamente los bosques de lenga se presentan como un mosaico de parches en distintos estados de desarrollo. Bosques en etapas sucesionales más avanzadas presentan una gran heterogeneidad espacial atribuida en buena parte a la dinámica de claros (Cavieres y Fajardo 2005). Además, condiciones diferenciales de sitio, es decir, altitud, tipo de suelo, posición topográfica, entre otras variables, podrían influir en estas diferencias estructurales (Pisano 1977, Kalin-Arroyo et al. 1996, Cuevas 2000).

Los resultados del presente estudio indican la existencia de un impacto directo del castor en el bosque ribereño debido al consumo por forrajeo preferencial de brinzales e individuos de diámetros menores a $25 \mathrm{~cm}$. Ello puede impactar negativamente el proceso de regeneración debido a que la destrucción directa de árboles jóvenes provocaría, al menos durante la presencia de los castores, una discontinuidad en el establecimiento de lenga en los ambientes ribereños. No obstante, las consecuencias de la interacción entre impactos directos e indirectos del castor, con otros factores ecológicos que intervienen en la dinámica de regeneración de lenga son aún poco predecibles. Además del impacto directo del castor por forrajeo, existen al 
menos dos impactos indirectos a considerar: el primero se refiere a la modificación de la profundidad de la napa freática y, por ende, las características del suelo (Baker y Hill 2003), lo que puede significar la muerte de árboles por inundación e impedir así la presencia de bancos de semillas. El segundo impacto indirecto del castor es la generación de meadows, en los cuales se impide la dinámica natural de regeneración del bosque ribereño (Anderson et al. 2006, Martínez.-Pastur et al. 2006). Existen también otros factores ecológicos relevantes que determinan la dinámica de regeneración de lenga, y que es necesario considerar como clave en el retardo o imposibilidad de establecimiento en las zonas ribereñas. Entre ellos están la variabilidad en la producción de semillas, estimada en 7-8 años (Cuevas 2000, 2002, Cavieres y Fajardo 2005), las bajas tasas de crecimiento anual (Aravena et al. 2002, González et al. 2007), la herbivoria del guanaco (Lama guanicoe Müller) en pequeños claros (Vázquez 2002, Cavieres y Fajardo 2005) y la potencial invasión de especies exóticas que podrían limitar el establecimiento de las especies nativas (Mella 1994, Anderson et al. 2006). Estudios futuros necesitan considerar estos factores y las relaciones existentes entre ellos a fin de entender, en el largo plazo, el impacto del castor en la regeneración de los bosques ribereños. Estudios dendrocronológicos que establezcan la fecha exacta de llegada del castor en cada sitio podrían ser muy útiles para entender la dinámica de colonización del castor. Al establecerse los tiempos de residencia y abandono de las castoreras, junto a las condiciones de sitio necesarias para su recolonización, se contribuirá a un mejor entendimiento de la dinámica y patrón de invasión del castor. Ello permitirá diseñar planes efectivos de control (zonas invadidas y con riesgo de recolonización), y construir así mapas de riesgo para las zonas que aún no han sido invadidas.

El presente estudio aporta nuevos antecedentes que permiten identificar a los brinzales como elementos clave en la selección de los sitios que el castor elige para invadir dentro de los bosques subantárticos. Al igual que en su ambiente original en el hemisferio norte, sitios con presencia del castor presentan como característica un diámetro de árboles y área basal menores. En el largo plazo, luego del establecimiento del castor y la consecuente muerte de los brinzales y árboles de menor diámetro por efecto de forrajeo, pueden ocurrir importantes cambios en la estructura y composición original del bosque ribereño.

\section{AGRADECIMIENTOS}

El presente trabajo fue financiado por la Iniciativa científica Milenio, Núcleo Forecos P04-065-F de MIDEPLAN y la Dirección de Investigación de la Universidad Austral de Chile (DID) proyecto SD-200771. Agradecemos las facilidades y apoyo logístico de la WCS (Wildlife Conservation Society) a través de Bárbara Saavedra,
Ricardo Muza y los guardaparques Antony Muñoz y Mauricio Chacón de la reserva Karukinka. También agradecemos a Christopher Anderson por su colaboración para llevar a cabo el trabajo de terreno y a tres revisores anónimos por sus sugerencias y comentarios que ayudaron a mejorar el manuscrito.

\section{REFERENCIAS}

Anderson CB, CR Griffith, AD Rosemond, R Rozzi, O Dollenz. 2006. The effects of invasive North American beavers on riparian plant communities in Cape Horn, Chile: do exotic beavers engineer differently in sub-Antarctic ecosystems? Biological Conservation 128: 467-474.

Aravena JC, A Lara, A Wolodarsky-Franke, R Villalba, E Cuq. 2002. Tree-ring growth patterns and temperature reconstruction from Nothofagus pumilio (Fagaceae) forests at the upper tree line of southern Chilean Patagonia. Revista Chilena de Historia Natural 75: 361-376.

Baker BW, EP Hill. 2003. Beaver (Castor canadensis). In Feldhamer GA, BC Thompson, JA Chapman eds. Wild Mammals of North America: Biology, Management, and Conservation. $2^{\text {nd }}$ Ed. Baltimore, Maryland, USA. The Johns Hopkins University Press. p. 288-310.

Barnes DM, AU Mallik. 1996. Use of woody plants in construction of beaver dams in northern Ontario. Canadian Journal of Zoology 74(17): 81-86.

Barnes DM, AU Mallik. 1997. Habitat factors influencing beaver dam establishment in a northern Ontario watershed. Journal of Wildlife Management 61: 1371-1377.

Bava JO, PM López-Bernal. 2006. Predicción del diámetro a la altura de pecho en función de las dimensiones del tocón de árboles de lenga (Nothofagus pumilio) en Tierra del Fuego, Argentina. Quebracho 13: 87-92.

Briones M, R Schlatter, A Wolodarsky, C Venegas. 2001. Clasificación ambiental para hábitat de Castor Canadensis (Khul 1820, Rodentia), de acuerdo a características de cuencas en un sector de Tierra del Fuego. Anales Instituto de la Patagonia, Serie Ciencias Naturales (Chile) 29: 75-93.

Cavieres L, A Fajardo. 2005. Browsing by guanaco (Lama guanicoe) on Nothofagus pumilio forest gaps in Tierra del Fuego, Chile. Forest Ecology and Management 204: 237-248.

Cottrell TR. 1995. Willow colonization of Rocky Mountain mires. Canadian Journal of Forest Research 25: 215-22.

Crooks JA. 2002. Characterizing ecosystem-level consequences of biological invasion: the role of ecosystem engineers. Oikos 97: 153-166.

Cruz P, P Honeyman, A Pezo, C Schulze. 2007. Análisis de crecimiento de árboles maduros de lenga (Nothofagus pumilio) en bosques de la XII Región, Chile. Bosque 28(1): 18-24.

Cuevas J. 2000. Tree recruitment at the Nothofagus pumilio alpine timberline in Tierra del Fuego, Chile. Journal of Ecology 88(5): 840-855.

Cuevas J. 2002. Episodic regeneration at the Nothofagus pumilio alpine timberline in Tierra del Fuego, Chile. Journal of Ecology 90: 52-60.

Donkor NT, JM Fryxell. 1999. Impact of beaver foraging on structure of lowland boreal forests of Algonquin Provincial Park, Ontario. Forest Ecology and Management 118: 83-92. 
Godoy JC. 1963. Fauna silvestre. Cons. Fed. Inves. Eval. Rec. Nat. Argentinos, Buenos Aires 8: 171-172.

González ME, C Donoso, P Ovalle, G Martínez-Pastur. 2007. Nothofagus pumilio (Poepp. et Endl.) Krasser. Lenga, roble blanco, leñar, roble de Tierra del Fuego. In Donoso C ed. Las especies arbóreas de los bosques templados de Chile y Argentina. Autoecología. Valdivia, Chile. Marisa Cúneo. p. 486-500.

Kalin-Arroyo M, C Donoso, R Murúa, E Pisano, R Schlatter, I Serey. 1996. Toward an ecologically sustainable forestry project, concepts, analysis and recommendations. Protecting Biodiversity and Ecosystem Processes in the Rio Condor Project - Tierra del Fuego. Santiago, Chile. Universidad de Chile, Departamento de Investigación y Desarrollo. $253 \mathrm{p}$.

Henríquez JC. 2004. Estado de la Turba esfangosa en Magallanes. In Blanco D, V de la Balze eds. Los turbales de la Patagonia: Bases para su inventario y la conservación de su biodiversidad. Publicación número 19. Buenos Aires, Argentina. Wetlands Internacional, p. 93-103.

Howard RJ, JS Larson. 1985. A stream habitat classification system for beaver. Journal of Wildlife Management 49: 19-25.

Ives RI. 1942. The beaver-meadow complex. Journal of Geomorphology 5: 191-203.

Johnston CA, RJ Naiman. 1990. Browse selection by beaver: Effects on riparian forest composition. Canadian Journal of Forest Research 20(10): 36-43.

Jones CG, JH Lawton, M Schachak. 1994. Organisms as ecosystem engineers. Oikos 69: 373-386.

Lathi S, M Helminen. 1974. The Beaver Castor fiber (L) and Castor canadensis (Kuhl) in Finland. Acta Theriologica 19(13): 177-189.

Lizarralde MS. 1993. Current status of the introduced beaver (Castor canadensis) population in Tierra del Fuego, Argentina. Ambio (22)6: 351-358.

Lizarralde M, J Escobar, G Deferrari. 2004. Invader species in Argentina: A review about the beaver (Castor canadensis) population on Tierra del Fuego ecosystem. Interciencia 29(7): 352-356.
Martínez-Pastur G, MV Lencinas, J Escobar, P Quiroga, L Malmierca, M Lizarralde. 2006. Understorey succession in Nothofagus forests in Tierra del Fuego (Argentina) affected by Castor canadensis. Applied Vegetation Science 9: 143-154.

Mella J. 1994. EIA. Estudios de Línea de Base Comisión Científica Proyecto Río Cóndor 94-14: Castores. 22 p.

Naiman RJ, G Pinay, CA Johnson, J Pastor. 1994. Beaver influences on the long-term biogeochemical characteristics of boreal forest drainage networks. Ecology 75(4): 905-921.

Pisano E. 1977. Fitogeografía de Fuego-Patagonia Chilena. I. Comunidades vegetales entre latitudes 52 y $56^{\circ} \mathrm{S}$. Anales del Instituto de la Patagonia 8: 121-250.

Rebertus AJ, TT Veblen. 1993. Structure and tree-fall gap dynamics of old-growth Nothofagus forests in Tierra del Fuego, Argentina. Journal of Vegetation Science 4: 641-654.

Rebertus AJ, T Kitzberger, TT Veblen, L Roovers. 1997. Blowdown history and landscape patterns in the Andes of Tierra del Fuego, Argentina. Ecology 78(3): 678-692.

Rubilar J. 1991. Respuesta de la Lenga (Nothofagus pumilio) ante intervenciones de raleo en la provincia de Última Esperanza. In Del Fierro P. 1998. Experiencia silvicultural del bosque nativo de Chile. Chile. 420 p.

Skewes O, F González, R Olave, A Ávila, V Vargas, P Paulsen, HE König. 2006. Abundance and distribution of American beaver, Castor canadensis (Kuhl 1820), in Tierra del Fuego and Navarino islands, Chile. Eur J Wildl Res. DOI 10.1007/ s10344-006-0038-2.

Terwilliger J, J Pastor. 1999. Small mammals, ectomycorrhizae, and conifer succession in beaver meadows. Oikos 85: 83-94.

Vázquez D. 2002. Multiple effects of introduced mammalian herbivores in a temperate forest. Biological Invasions 4: 175-191.

Wallem P, CG Jones, PA Marquet, FM Jaksic. 2007. Identificación de los mecanismos subyacentes a la invasión de Castor canadensis (Rodentia) en el archipiélago de Tierra del Fuego, Chile. Revista Chilena de Historia Natural 80: 309-325.

Wright JP, CG Jones, AS Flecker. 2002. An ecosystem engineer, the beaver, increases species richness at the landscape scale. Oecologia 132: 96-101. 